\title{
Thioredoxin - a magic bullet or a double-edged sword for mammalian aging?
}

\author{
Yuji Ikeno ${ }^{\mathrm{a}, \mathrm{b}, \mathrm{c}^{*}}$ \\ ${ }^{a}$ Barshop Institute for Longevity and Aging Studies, San Antonio, TX 78229, USA. \\ ${ }^{b}$ Department of Pathology, The University of Texas Health Science Center at San Antonio, San Antonio, TX 78229, USA. \\ ${ }^{c}$ Geriatric Research and Education Center, Audie L. Murphy VA Hospital South Texas Veterans Health Care System, San \\ Antonio, TX 78229, USA.
}

\begin{abstract}
After the discovery of thioredoxin as a reductant for many important enzymes in the early 1960s, biological roles of thioredoxin in pathophysiology have been examined using various species and experimental models, e.g., yeast, invertebrates, rodents, and humans. A large number of studies demonstrated that thioredoxin plays an essential role to maintain a reduced cellular environment and possesses many beneficial effects by maintaining cellular/organ functions and against diseases. However, an important question that remains to be answered is whether thioredoxin could attenuate aging by reducing oxidative damage and changing cellular redox state, which alters redox-sensitive signaling pathways. To address this important question, we have been conducting aging studies with transgenic and knockout mice, and transgenic rats to examine whether the upregulation or downregulation of thioredoxin alters lifespan and age-related pathology. Aging studies conducted by our laboratory and others revealed that the roles of thioredoxin on pathophysiology seem to be more complex than our initial expectations as a potential magic bullet to solve the issues with age. Recent studies indicate that thioredoxin could have both beneficial and potentially deleterious effects on aging and age-related diseases. To critically evaluate the biological effects of thioredoxin on aging and age-related diseases, studies require further consideration to assess additional factors, e.g. levels of thioredoxin in different cellular compartments, different effects in each cell/tissue/organ, physiological aging vs. pathology, and/or at different life stages.

Keywords: Thioredoxin, transgenic mouse, knockout mouse, oxidative stress, cancer, aging
\end{abstract}

\section{Thioredoxin}

Since its discovery as the reductant for various enzymes in the early $1960 \mathrm{~s}$, biological roles of thioredoxin (Trx), which is a small protein $(12 \mathrm{kDa})$ with two redox-active cysteine residues in the active center (Cys-Gly-Pro-Cys), have been investigated in various species. Isoforms of Trx were identified in E. coli, yeast, and mammals. In humans, Trx mainly localizes in the cytosol (Trx1) and in mitochondrial (Trx2). Trx plays an important role to maintain cellular functions. For example, Trx is a hydrogen

*Corresponding author: Yuji Ikeno, MD. PhD.

Mailing address: Barshop Institute for Longevity and Aging Studies, Department of Pathology, The University of Texas Health Science Center at San Antonio, 4939 Charles Katz Dr., San Antonio, TX 78229, USA.

E-mail: ikeno@uthscsa.edu

Received: 28 May 2021 / Accepted: 29 May 2021 donor for ribonucleotide reductase, peroxiredoxin (Prx), and methionine sulfoxide (MetO) reductase, which is essential for DNA synthesis or protection against oxidative stress. In addition, Trx directly and efficiently reduces disulfides and maintains cysteine residues in a reduced state in proteins through dithiol-disulfide exchange reactions. Through these reactions, Trx plays a very important role to maintain a reduced cellular environment, and unlike other major antioxidant enzymes, Trx's ability to change the redox state has another important role to regulate transcription factors' DNA binding activity and downstream genes expression. Because of these diverse effects on cellular physiology, Trx drew much attention in its roles in pathophysiology including aging.

\section{Aging studies with mice upregulating or down- regulating Trx1 or Trx2}

The first aging study using transgenic (Tg) mice overexpressing Trx1 was conducted by Yodoi and colleagues in 
1999. This study demonstrated that Trx 1 transgenic mice showed increased resistance against brain ischemic injury, reduced protein oxidation, and an extension of lifespan compared to control mice [1-3]. These results were very exciting because of Trx's potential as a magic bullet to attenuate aging and some common age-related disorders, e.g., ischemic injury in the tissues, by increased resistance to oxidative stress. To further explore this possibility, our laboratory conducted the first systematic aging studies using transgenic/knockout mice upregulating or downregulating Trx1 or Trx2.

Our initial aging study with Trx 1 transgenic mice (obtained from Dr. Yodoi's group) showed increased resistance against oxidative stress both in vitro and in vivo, and less oxidative damage to macromolecules [4]. The survival study showed a significant increase of lifespan only in the earlier part of the life of male Trx 1 transgenic mice compared to the wild-type mice, which was associated with a reduced incidence of inflammatory lesions in the lung [4]. To confirm these results, another line of Trx1 transgenic mice was generated by our laboratory and a survival study was conducted, which also showed similar results, i.e., Trx 1 overexpression showed benefits only in the earlier part of lifespan [5]. Pathological analyses of Trx1 transgenic mice showed accelerated tumor formation in these mice especially in the latter part of the lifespan, while there were some beneficial effects against non-neoplastic lesions in the earlier part of lifespan [4].

Effects of mitochondrial Trx on aging were tested using mice overexpressing Trx 2 . Trx 2 transgenic mice showed several important biological effects shown by another laboratory and ours [6-8]. The survival study with Trx2 transgenic mice also showed a slight extension of lifespan only in the earlier part of life with an indication of accelerated tumor formation, which is similar to the effects of Trx1 overexpression [8].

Trx downregulation and their biological effects were tested using heterozygous knockout (KO) mice for Trx 1 or $\operatorname{Trx} 2$ because the null mice for $\operatorname{Trx} 1$ or $\operatorname{Trx} 2$ are embryonic lethal $[9,10]$. Although downregulation of Trx 1 or Trx2 showed some interesting biological effects, e.g., enhanced apoptosis, impaired mitochondrial function with increased ROS production, etc., no notable effects on survival curves or pathology were observed [11-13].

\section{Synergistic effects of upregulating or downreg- ulating Trx 1 and Trx 2 on aging}

As described above, overexpression or downregulation of thioredoxin only in one compartment of the cell, i.e., either only in cytosol or mitochondria, did not show a strong impact on aging. The next question we addressed was whether synergistic upregulation or downregulation of Trx 1 and Trx2 attenuates aging and age-related diseases, and survival studies were conducted using Trx1Tg $\mathrm{x}$ Trx 2 Tg and Trx1 KO x Trx2 KO mice.

The results with Trx1 Tg $\mathrm{x} \operatorname{Trx} 2 \mathrm{Tg}$ mice were opposite to our expectations, in which male Trx $1 \operatorname{Tg} x$ Trx 2 Tg mice lived significantly shorter than WT littermates [14]. Interestingly, the shorter lifespan was accompanied by accelerated cancer growth [14]. On the other hand, our ongoing study shows that Trx $1 \mathrm{KO} \times \mathrm{Trx} 2 \mathrm{KO}$ mice attenuate cancer development with a modest increase in lifespan compared to WT mice. These results convincingly suggest that reduced Trx in both the cytosol and mitochondria provides benefits to aging animals by suppressing cancer development through several underlying mechanisms unique to biological effects by Trx.

\section{Is thioredoxin a double-edged sword for mam- malian aging?}

Based on our observations with Trx1 and Trx 2 transgenic and heterozygous knockout mice, the effects of Trx on aging pathophysiology we have learned are: 1) synergistic upregulation or downregulation of $\operatorname{Trx}$ has a significant impact on cancer development, which is accompanied by some changes in lifespan, i.e., a significant reduction in lifespan by synergistic upregulation and modest extension by synergistic downregulation; and 2) Trx1 upregulation provides protective effects against oxidative stress in vitro and in vivo, suppresses inflammatory lesions in lung (acidophilic macrophage pneumonia), and has a slight extension of the earlier part of lifespan. These results could suggest that changes in Trx levels show both beneficial and deleterious effects, i.e., a double-edged sword, which could be further affected by additional factors. These complexed roles of Trx could be affected by: 1) young vs. old; 2) cell/tissue/organ; 3) physiology and pathology (cancer, degenerative disorder or inflammation, etc.); and 4) changes only in one cellular compartment or synergistic changes.

To critically evaluate the biological effects of thioredoxin on aging and age-related diseases, further studies to seek the underlying mechanisms: 1) why synergistic downregulation attenuated cancer development; 2) why upregulation of Trx1 suppressed inflammatory lesions in the lung in young animals, are required and those studies will lead us to identify new interventions for the prevention/therapy of age-related diseases and to extend healthspan in humans.

\section{Declarations}

Acknowledgments: This research was supported by the VA Merit Review Grant 1 I01BX001023 from the Biomedical Laboratory Research \& Development Service of the Veteran's Affairs Office of Research and Development (Y.I.), and NIH Grant AG13319 (Y.I.).

Conflict of interest: The author declared that there are no conflicts of interest. 


\section{References}

1. Takagi Y, Mitsui A, Nishiyama A, et al. Overexpression of thioredoxin in transgenic mice attenuates focal ischemic brain damage. Proceedings of the National Academy of Sciences, 1999, 96(7): 4131-4136.

2. Mitsui A, Hamuro J, Nakamura $\mathrm{H}$, et al. Overexpression of human thioredoxin in transgenic mice controls oxidative stress and life span. Antioxidants and Redox Signaling, 2002, 4(4): 693-696.

3. Nakamura H, Mitsui A, Yodoi J. Thioredoxin overexpression in transgenic mice. Methods in enzymology, 2002, 347: 436-440.

4. Pérez V I, Cortez L A, Lew C M, et al. Thioredoxin 1 overexpression extends mainly the earlier part of life span in mice. Journals of Gerontology Series A: Biomedical Sciences and Medical Sciences, 2011, 66(12): 1286-1299.

5. Flores L C, Roman M G, Cunningham G M, et al. Continuous overexpression of thioredoxin 1 enhances cancer development and does not extend maximum lifespan in male C57BL/6 mice. Pathobiology of Aging \& AgeRelated Diseases, 2018, 8(1): 1533754.

6. Widder J D, Fraccarollo D, Galuppo P, et al. Attenuation of angiotensin II-induced vascular dysfunction and hypertension by overexpression of thioredoxin 2. Hypertension, 2009, 54(2): 338-344.

7. Zhang H, Luo Y, Zhang W, et al. Endothelial-specific expression of mitochondrial thioredoxin improves endothelial cell function and reduces atherosclerotic lesions. The American journal of pathology, 2007, 170(3): 11081120.
8. Roman M G, Flores L C, Cunningham G M, et al. Thioredoxin overexpression in mitochondria showed minimum effects on aging and age-related diseases in male C57BL/6 mice. Aging Pathobiology and Therapeutics, 2020, 2(1): 20-31.

9. Matsui M, Oshima M, Oshima $\mathrm{H}$, et al. Early embryonic lethality caused by targeted disruption of the mouse thioredoxin gene. Developmental biology, 1996, 178(1): 179-185.

10. Nonn L, Williams R R, Erickson R P, et al. The absence of mitochondrial thioredoxin 2 causes massive apoptosis, exencephaly, and early embryonic lethality in homozygous mice. Molecular and cellular biology, 2003, 23(3): 916.

11. Pérez V I, Lew C M, Cortez L A, et al. Thioredoxin 2 haploinsufficiency in mice results in impaired mitochondrial function and increased oxidative stress. Free Radical Biology and Medicine, 2008, 44(5): 882-892.

12. Pérez V I, Bokov A, Van Remmen H, et al. Is the oxidative stress theory of aging dead?. Biochimica et Biophysica Acta (BBA)-General Subjects, 2009, 1790(10): 10051014.

13. Roman M G, Flores L C, Cunningham G M, et al. Thioredoxin and aging: What have we learned from the survival studies?. Aging Pathobiology and Therapeutics, 2020, 2(3): 126-133.

14. Cunningham G M, Flores L C, Roman M G, et al. Thioredoxin overexpression in both the cytosol and mitochondria accelerates age-related disease and shortens lifespan in male C57BL/6 mice. Geroscience, 2018, 40(5): 453-468.

Cite this article as: Yuji I. Thioredoxin - a magic bullet or a double-edged sword for mammalian aging?[J]. Aging Pathobiology and Therapeutics, 2021, 3(2): 17-19. 